\title{
Lysyl oxidase is involved in synovial hyperplasia and angiogenesis in rats with collagen-induced arthritis
}

\author{
FAN WANG ${ }^{1}$, JUAN WAN $^{1}$, QIUYAN LI $^{2}$, MINGZHU ZHANG ${ }^{1}$, QIAOFENG WAN ${ }^{2}$, \\ CHEN JI $^{1}$, HAIBO LI ${ }^{1}$, RONGQING LIU ${ }^{1}$ and MEI HAN ${ }^{2}$ \\ ${ }^{1}$ Department of Rheumatology and Immunology, The General Hospital of Ningxia Medical University; \\ ${ }^{2}$ Department of Pathogenic Biology and Immunology, Ningxia Medical University, Yinchuan, Ningxia 750004, P.R. China
}

Received November 1, 2016; Accepted June 13, 2017

DOI: $10.3892 / \mathrm{mmr} .2017 .7436$

\begin{abstract}
Lysyl oxidase (LOX) serves an important role in remodeling the extracellular matrix and angiogenesis in various types of cancer; however, whether LOX is involved in the pathogenesis of rheumatoid arthritis remains unknown. In order to investigate this in the present study, $\beta$-aminopropionitrile, an inhibitor of LOX, was injected intraperitoneally into rats with type II collagen-induced arthritis (CIA). Subsequently, synovial hyperplasia was examined by hematoxyl in and eosin staining, and the microvascular density (MVD) and expression levels of LOX, matrix metalloproteinase (MMP)-2 and MMP-9 in the synovial membrane and fluid were determined by immunohistochemistry and ELISA, respectively. The enzyme activity of LOX was evaluated by the Amplex Red Hydrogen Peroxide method. The results demonstrated an increased amount of rough synovial membranes, higher MVD in these membranes and more synovial cell layers in CIA rats compared with in the control rats. In addition, higher enzymatic activity of LOX and higher expression levels of MMP-2 and MMP-9 were revealed in CIA rats compared with in the control rats. Notably, $\beta$-aminopropionitrile inhibited paw swelling and the decreased the arthritis index, the MVD in the synovial membranes and the expression levels of MMP-2 and MMP-9. Furthermore, the expression level of LOX in the synovial membranes was positively associated with the MVD and the expression levels of MMP-2 and MMP-9, suggesting that LOX promotes synovial hyperplasia and angiogenesis and that LOX may be a potential therapeutic target for rheumatoid arthritis.
\end{abstract}

Correspondence to: Dr Rongqing Liu, Department of Rheumatology and Immunology, The General Hospital of Ningxia Medical University, 804 Shengli Street, Yinchuan, Ningxia 750004, P.R. China E-mail: liurongqing1964@163.com

Professor Mei Han, Department of Pathogenic Biology and Immunology, Ningxia Medical University, 1160 Shengli Street, Yinchuan, Ningxia 750004, P.R. China

E-mail: hanmei0708@126.com

Key words: lysyl oxidase, collagen-induced arthritis, rheumatoid arthritis, matrix metalloproteinase-2, matrix metalloproteinase-9

\section{Introduction}

Rheumatoid arthritis (RA) is a systemic autoimmune disease characterized by chronic, progressive and invasive arthrosynovitis (1). RA affects $0.5-1 \%$ of the adult population (2). Its pathological features include persistent synovitis, abnormal synovial hyperplasia, increased angiogenesis and pannus formation. RA gradually expands into the articular surface and articular cartilage to induce the progressive destruction of cartilage and bones, eventually resulting in joint deformity $(3,4)$. There have been many recent and relevant studies on RA; however, its pathogenic mechanism has not yet been elucidated.

Lysyl oxidase (LOX), an extracellular copper-dependent amine oxidase, participates in the catalysis of cross-links between the lysine residues of collagen and elastin in the extracellular matrix, and is important in the initial stage of the conversion of soluble collagen and elastin monomers into insoluble fibers. LOX is involved in numerous cellular physiological and pathological processes including extracellular matrix formation, cell proliferation, chemotaxis, inflammation, angiogenesis and tumor formation (5). Abnormal LOX expression is associated with the occurrence and development of various diseases. Decreased expression, decreased activity and a lack of LOX are associated with cutis laxa, emphysema and uterine prolapse $(6,7)$, whereas increased LOX expression is associated with scleroderma $(8)$, cirrhosis $(9,10)$ and tumor metastasis $(11,12)$. A previous study of the authors indicated that high concentrations of LOX are present in the synovial membrane and synovial fluid of patients with RA (13); however, the role of LOX in joint diseases associated with RA remains unclear.

The type II collagen-induced arthritis (CIA) model is currently the most commonly used animal model for RA studies, as it has immunological, pathological and arthritic presentations similar to those observed in RA in humans $(14,15)$. In the present study, a rat CIA model was established and $\beta$-aminopropionitrile (BAPN) was used to inhibit LOX activity $(16,17)$ in order to observe synovial hyperplasia and angiogenesis, and to investigate the role and mechanism of LOX in arthritic diseases of rats. The present study aimed to provide theoretical bases for further studies investigating pathogenic mechanism underlying RA and novel targets for clinical treatment. 


\section{Materials and methods}

Animals. A total of 30 6-8-week-old healthy male Sprague Dawley rats (SPF grade) with body weights of 180-200 g were purchased from Shanghai Sino-British SIPPR/BK Laboratory Animal Co., Ltd. [Shanghai, China; permit no: SCXK (Hu) 2013-0016]. Rats were housed in separate cages and were fed a standard diet every day. Following adaptive feeding under the conditions of a 12:12 $\mathrm{h}$ light:dark cycle, with a temperature of $22 \pm 2^{\circ} \mathrm{C}$, and $55 \pm 5 \%$ humidity for 1 week, experiments were performed in accordance with the Guidelines for the Care and Use of Laboratory Animals. The present study was approved by the Ethics Committee of the General Hospital of Ningxia Medical University (Yinchuan, China; registration no. 2016-230).

Establishment of the CIA model and drug administration. SD rats were randomly divided into a control group, model group and intervention group ( $\mathrm{n}=10 /$ group). For rats in the model group, $0.2 \mathrm{ml}$ bovine collagen II (Sigma-Aldrich; Merck KGaA, Darmstadt, Germany) emulsified by complete Freund's adjuvant (Sigma-Aldrich; Merck KGaA) was intradermally injected into the back, tail and footpad; a booster was administered after 7 days using the same method, in the same locations, and at the same dose. The model establishment method for rats in the intervention group was the same as that in the model group; in addition, BAPN (Sigma-Aldrich; Merck KGaA) was intraperitoneally injected (100 mg/kg/days) for 40 days to inhibit LOX activity. Rats in the control group were injected with an equal volume of normal saline using the same method and at the same locations as in the model group.

Assessment of the arthritis index (AI). From day 4 after the booster administration, the conditions and extent of joint redness and swelling were evaluated every 4 days using the AI scoring method of the left hind paw joint of each rat. This was determined using a 5 -grade scoring method: 0 , normal, without any macroscopic signs of arthritis; 1 , mild redness and swelling of the toe joint; 2 , moderate redness and swelling of the toe joint; 3 , severe redness and swelling of the ankle; and 4 , extreme severe redness, swelling and deformation of the ankle joint, including complete paw swelling and inability to bend the joint and walk $(18,19)$. Rats with an AI score of $\geq 2$ were selected for use in the subsequent experiments.

Evaluation of paw edema. The assessment of paw edema was performed one day prior to the first injection and once every 4 days following the booster injection. The left hind paw volume of each rat was determined using the drainage volume method, as follows. A line was drawn as a marker at the salient section of the left hind paw ankle joint of each rat. A specific measuring cylinder was filled with water and the paw was inserted to allow the water surface to reach the marked level of the labeled ankle. A beaker was used to capture the volume of the water displaced, which determined the paw volume in $\mathrm{ml}$. Each paw evaluation was repeated five times and the mean value was recorded.

Sample collection. At the end of experiment, rats were anesthetized with chloral hydrate $(350 \mathrm{mg} / \mathrm{kg}$; intraperitoneal) and sacrificed by cervical dislocation. Blood, synovial fluid and synovial membrane from the knee joint were collected. The collected blood samples were incubated at room temperature for $2 \mathrm{~h}$ and then centrifuged at 2,000 $\mathrm{xg}$ for $20 \mathrm{~min}$ to obtain the serum. The synovial fluids were centrifuged in order to obtain the supernatants. Serum and supernatant samples were divided into aliquots and frozen at $-80^{\circ} \mathrm{C}$ for subsequent use. Synovial membranes were washed with sterile normal saline, placed in a $0.5 \mathrm{ml}$ tube, labeled and stored at $-80^{\circ} \mathrm{C}$.

Hematoxylin and eosin $(H \& E)$ staining. Rat synovial membranes were fixed using $4 \%$ paraformaldehyde, dehydrated in graded alcohol, cleared with xylene, embedded in paraffin and sectioned. H\&E staining was performed and hyperplasia of the synovial membrane was observed under a light microscope. For each membrane section, five areas were selected randomly and images were captured using a low-power lens (magnification, x100).

Inspection of microvascular density (MVD). Synovial membrane sections were blocked in $10 \%$ fetal bovine serum at room temperature for $30 \mathrm{~min}$ and then incubated with rabbit anti-rat CD34 monoclonal antibodies (1:200 dilution, cat. no. ab81289; Abcam, Cambridge, MA, USA) at $4^{\circ} \mathrm{C}$ overnight. Following washing, the membrane sections were processed with rabbit horseradish peroxidase (HRP)-polymer kit (cat. no. PV-6001; ZSGB-BIO, Beijing, China) at room temperature, developed using 3,3'-diaminobenzidine (DAB) for $4 \mathrm{~min}$ and mounted on slides. MVD was determined by evaluating the number of CD34-stained vessels in the membrane samples. In brief, the five hot spot areas with the highest numbers of microvessels were selected from each membrane section using a low-power lens (magnification, x100), and numbers of microvessels in each of these areas were determined using a high-power lens (magnification, x400). The mean microvessel number represented the MVD in the synovial membrane.

Immunohistochemical (IHC) analysis. Synovial membrane sections were blocked and then incubated with rabbit anti-rat LOX polyclonal antibodies (1:100 dilution; cat. no. ab31238; Abcam), rabbit anti-rat MMP-2 polyclonal antibodies (1:400 dilution; cat. no. ab37150; Abcam) or mouse anti-rat MMP-9 monoclonal antibodies (1:500 dilution; cat. no. ab38898; Abcam) at $4^{\circ} \mathrm{C}$ overnight. Following washing, the membrane sections were processed with rabbit HRP-polymer kit (cat. no. PV-6001; ZSGB-BIO, Beijing, China), developed using DAB for 4 min, cleared and mounted on slides. Under a light microscope, five or more hot spots in each section were observed. Images were captured using DP controller software. Image-Pro Plus version 6.0 software (Media Cybernetics, Inc., Rockville, MD, USA) was used for image analysis to determine the positively stained surface areas and the integrated optical densities (IODs) of captured images. The relative expression level was calculated using the average optical density (AOD): $\mathrm{AOD}=\mathrm{IOD} /$ positive area.

ELISA. LOX, MMP-2 and MMP-9 concentrations in the synovial fluid and serum were detected using rat ELISA kits (cat nos. SEC580Ra, SEA100Ra and SEA553Ra), according to the manufacturer's instructions (Cloud-Clone Corp., Houston, 
TX, USA). Synovial fluid was diluted at 1:10, and serum was diluted at 1:5. The optical density values were used to calculate the concentrations of LOX, MMP-2 and MMP-9 in all of the samples. The concentration was multiplied by the dilution fold in order to obtain the actual concentration.

Amplex Red method.An Amplex Red reagent kit was purchased from Abcam (cat. no. ab112139; Cambridge, MA, USA) and used to evaluate LOX activities in the rat serum and synovial fluid. All experimental procedures were performed according to the manufacturer's protocol. Synovial fluid and serum were diluted at 1:5. The diluted serum and synovial fluid samples were divided into the experimental group and the parallel group. All wells in the parallel group were supplemented with $0.3 \mathrm{mM}$ BAPN to inhibit the activity of LOX. Various concentrations of $\mathrm{H}_{2} \mathrm{O}_{2}$ were used as the standards of oxidation in order to plot a standard curve and obtain the corresponding calculation formula. Finally, the oxidation activity value in the parallel group was subtracted from the oxidation activity value in the experimental group to determine the oxidation activity value of LOX in the samples.

Statistical analysis. Data are presented as the mean \pm standard deviation. Statistical analyses were performed using SPSS statistical software (version, 20.0; IBM SPSS, Armonk, NY, USA). Normally distributed continuous variables were compared using one-way analysis of variance. Non-normally distributed data were compared using nonparametric tests, including the Mann-Whitney U test or the Kolmogorov-Smirnov test. Pearson correlation analysis was performed to analyze the correlation between LOX expression level and MVD. $\mathrm{P}<0.05$ was considered to indicate a statistically significant difference.

\section{Results}

Inhibiting LOX with BAPN decreased the AI scores in CIA rats. AIs were determined on days 4, 8, 12, 16, 20, 24 and 28 following the booster immunization with collagen. Rats in the control group demonstrated no macroscopic signs of arthritis. AI scores in the model group and the intervention group were higher compared with those in the control group $(\mathrm{P}<0.05)$, and the scores in the intervention group were lower compared with those in the model group on days 16 , 20 and $24(\mathrm{P}<0.05$; Fig. 1). The redness and swelling of the toe joint was markedly ameliorated following inhibition of LOX by BAPN. An AI score of $\geq 2$ for each rat in the model and intervention group was considered to indicate successful model establishment.

Inhibiting LOX with BAPN attenuated the hind paw swelling in CIA rats. Prior to the first collagen injection (day 0), no significant hind paw swelling in any of the rats of the three experimental groups was observed $(\mathrm{P}>0.05)$. At day 4 following the collagen booster, significantly increased levels of hind paw swelling were observed in rats of the model and intervention groups compared with those in the control group $(\mathrm{P}<0.05)$, however, the paw swelling in rats of the intervention group was decreased on days 16, 20 and 24 compared with in the model group $(\mathrm{P}<0.05$; Fig. 2$)$.

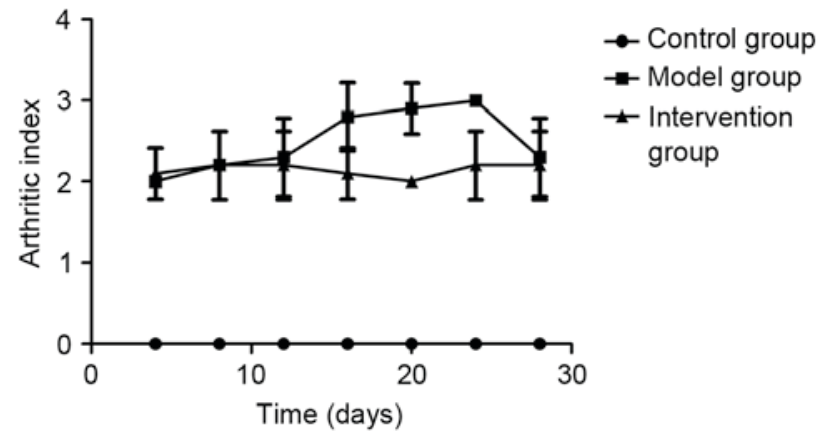

Figure 1. Dynamic observation of AI scores. AI scores of the 10 rats in the control group were 0 . AI scores were significantly higher for the CIA model rats $(\mathrm{P}<0.05)$. Following inhibition of LOX by BAPN (intervention group), decreased AI scores were observed on days 16-24. Data are presented as the mean \pm standard deviation. $\mathrm{P}<0.05$ vs. model group. AI, arthritis index; CIA, collagen-induced arthritis; LOX, lysyl oxidase; BAPN, $\beta$-aminopropionitrile.

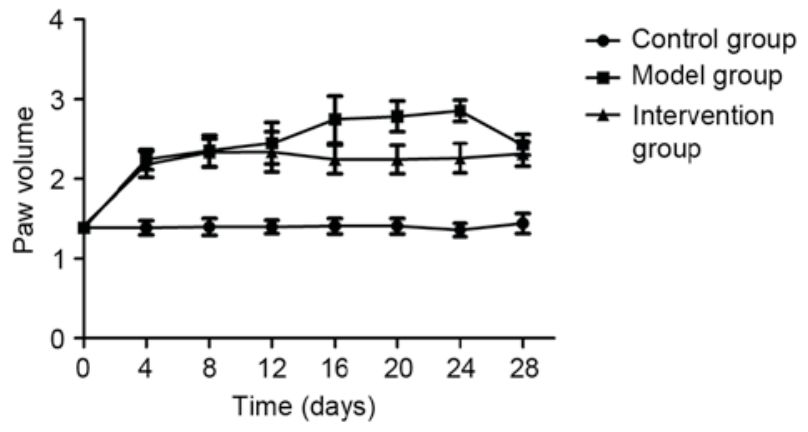

Figure 2. Dynamic changes in paw swelling. Compared with the rats in the control group, paw volume was significantly increased in the CIA model rats $(\mathrm{P}<0.05)$. Following inhibition of LOX by BAPN (intervention group), paw edema was decreased on days 16-24 $(\mathrm{P}<0.05)$. Data are presented as the mean \pm standard deviation. CIA, collagen-induced arthritis; LOX, lysyl oxidase; BAPN, $\beta$-aminopropionitrile.

Inhibiting LOX with BAPN decreased synovial hyperplasia in CIA rats. The synovial membrane surfaces of rats in the control group were smooth and synovial cells were arranged in a uniform manner as observed by light microscopy following H\&E staining. Following a longer inflammation induction in the model and intervention groups, rough synovial tissue surfaces and thickening of synovial tissues were observed; the number of synovial cell layers increased and the layers exhibited a disorderly arrangement, as presented in Fig. 3.

Inhibiting LOX with BAPN increases MVD in CIA rats. CD34-positive staining, indicated by a yellow-brown color, was localized in the cytoplasm of vascular endothelial cells. CD34-positive cells surrounded a lumen-like structure, forming microvessels (Fig. 4). The MVDs were 13.44 \pm 2.94 in the model group and $11.22 \pm 1.67$ in the intervention group, and were higher compared with that in the control group (9.10 $\pm 1.94 ; \mathrm{P}<0.05)$. The MVD in the intervention group was lower compared with that in the model group $(\mathrm{P}<0.05)$.

Expressions levels of LOX, MMP-2 and MMP-9 in synovial membrane of rats. The IHC results indicated that LOX expression in the rat synovial membrane was primarily distributed in the cytoplasm of synovial cells and the extracellular 


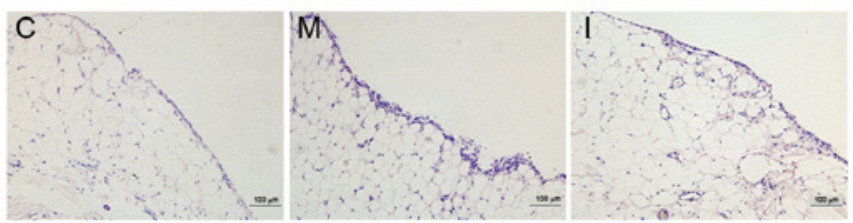

Figure 3. Synovial hyperplasia of rats following H\&E staining. In the control group (C), the structure of the synovial membrane was clear and smooth, without hyperplasia. In the model group (M), the structure of the synovial tissue demonstrated disorder and the layers of synovial cells were more randomly arranged. In the intervention group (I), synovial hyperplasia was reduced compared with that in the model group. H\&E, hematoxylin and eosin.

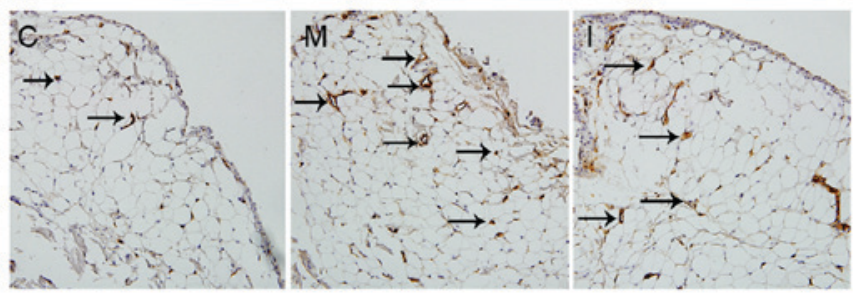

Figure 4. MVD in synovial tissues of rats. MVD was determined by CD34 immunohistochemical staining. Black arrows indicate the microvessels, surrounded by CD34-positive cells, which formed a lumen-like structure. Magnification, $x 400$. Low density of microvessels was observed in the control group (C). High density of microvessels was observed in the model group (M). MVD in the intervention group (I) was decreased compared with that in the model group. MVD, microvascular density; CD34, cluster of differentiation 34.

matrix (Fig. 5A). MMP-2 and MMP-9 were also primarily distributed in the cytoplasm of synovial cells (Fig. 5B and C). The AODs of LOX, MMP-2 and MMP-9 in rat synovial membrane of the model group were higher compared with those in the control group and the intervention group $(\mathrm{P}<0.05)$, whereas the AODs of LOX, MMP-2 and MMP-9 in the intervention group were lower compared with those in the control group $(\mathrm{P}<0.05$; Fig. 5D).

Comparison of LOX, MMP-2 and MMP-9 expression levels in the synovial fluid and serum. ELISA results demonstrated that the concentrations of LOX, MMP-2 and MMP-9 in the synovial fluid and serum of rats from the model group were significantly higher compared with those in the control and intervention groups $(\mathrm{P}<0.05)$. Concentrations of LOX, MMP-2 and MMP-9 in the control group were significantly higher compared with that in the intervention group $(\mathrm{P}<0.05$; Table I).

Comparison of LOX enzyme activity in the serum and synovial fluid. The enzyme activity of LOX in the model group was significantly higher compared with that in the control and intervention groups $(\mathrm{P}<0.05)$, and the LOX activity in rats of the control group was higher compared with that in the intervention group $(\mathrm{P}<0.05$; Table I).

Correlation of LOX expression levels with MVD, MMP-2 and MMP-9 in the synovial membrane. Pearson correlation analysis revealed that the expression level of LOX in the synovial membrane was positively correlated with MVD $(r=0.253$; $\mathrm{P}<0.05)$, MMP-2 ( $\mathrm{r}=0.741 ; \mathrm{P}<0.01)$ and MMP-9 ( $\mathrm{r}=0.644$; $\mathrm{P}<0.01)$.

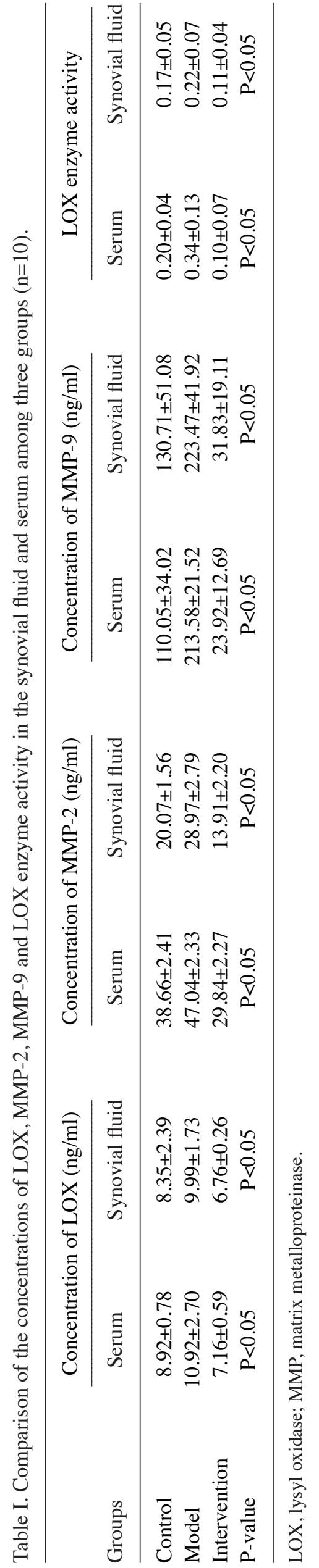



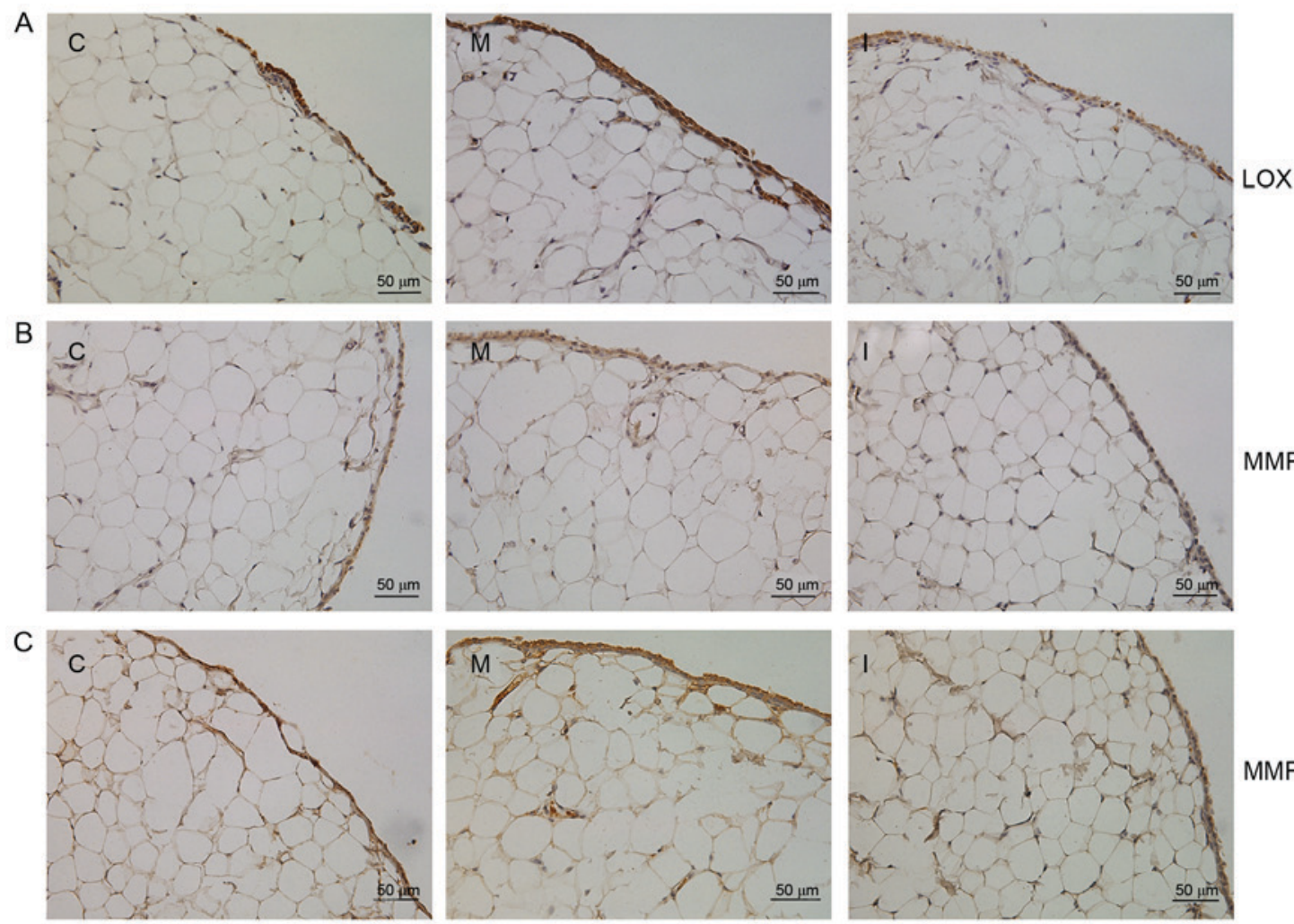

MMP-2
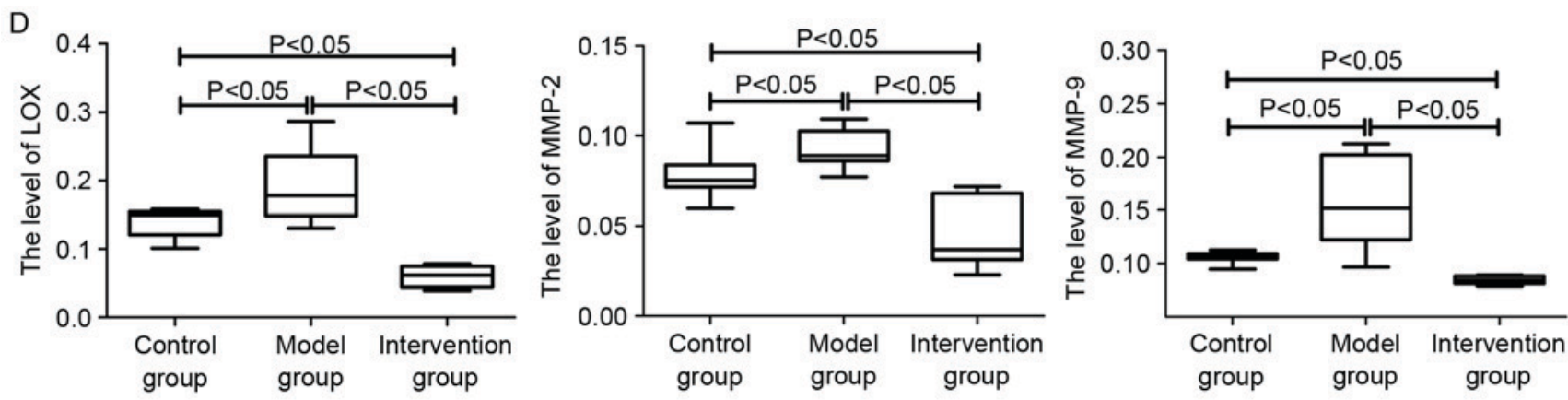

Figure 5. Expressions levels of (A) LOX, (B) MMP-2 and (C) MMP-9 in rats of the synovial membrane of the control group (C), model group (M) and intervention group (I), as determined by immunohistochemical staining. Following establishment of the CIA model, the expressions levels of LOX, MMP-2 and MMP-9 in the synovial membranes were increased compared with those in the control group. However, following inhibition of LOX with BAPN, the expression levels of MMP-2 and MMP-9 were decreased significantly compared with those in (D) the model group. Data are presented as the mean \pm standard deviation. LOX, lysyl oxidase; MMP, matrix metalloproteinase; CIA, collagen-induced arthritis; BAPN, $\beta$-aminopropionitrile.

\section{Discussion}

The pathogenic mechanism underlying RA is complicated. Genetic, environmental, and immunological factors affect its occurrence and development $(20,21)$. Increasing evidence indicates that various immune cells (including $\mathrm{T}$ lymphocytes, B lymphocytes, and synovial fibroblasts), cytokines (including tumor necrosis factor- $\alpha$ and interleukin-6), and enzymes (MMPs) are involved in the mechanisms underlying RA $(22,23)$. However, whether LOX affects RA, and the potential underlying mechanism of action of LOX remains unclear.

In the present study, CIA rats were selected as an experimental animal model. During the process of model establishment, rats in the model group and the intervention group exhibited hind paw redness and swelling from day 2 following the first injection. On day 24 following administration of the collagen booster, the hind paw and ankle joint swelling was most severe. At all evaluation time-points, the
AI and joint swelling of rats in the model and intervention groups were significantly higher compared with those in rats of the control group; these findings are in accordance with the disease pattern of the type II collagen-induced CIA model. H\&E staining of the synovial membrane demonstrated that rats in the model and intervention groups had rough and thickened synovial tissue surfaces and increased numbers of disordered synovial cell layers. In addition, the MVD in the synovial membrane was higher compared with that in the control group. These results confirmed that the model was successfully established.

LOX is an oxidase secreted by numerous types of cells and is a key enzyme involved in the cross-linking of collagen and elastin in extracellular matrix. LOX participates in the stabilization and remodeling of the extracellular matrix (24). LOX has a $50 \mathrm{kDa}$ zymogene form and a $32 \mathrm{kDa}$ active form. The active form of LOX is secreted into the serum and bodily fluids. The results of the present study demonstrated that the 
concentrations of LOX in the synovial membrane, synovial fluid and serum of rats in the model group were significantly higher compared with those in the control group. The enzymatic activity of LOX in the synovial fluid and serum of the model group was higher compared with that in the control group; in addition, the concentration of LOX was positively correlated with the MVD in the synovial membranes. These results suggested that LOX may be involved in diseases of the joints and angiogenesis in rats of the model group.

BAPN is an irreversible inhibitor of LOX. Following LOX inhibition by BAPN in rats of the intervention group, the concentrations and activities of LOX in the serum, synovial fluid and synovial membrane were significantly decreased compared with those in the model and control groups. These results revealed that BAPN inhibited but did not completely suppress LOX in rats. In the present study, the AI, hind paw swelling, synovial hyperplasia and MVD of rats in the intervention group were lower compared with those in rats of the CIA model group. These results suggested that the suppression of LOX may reduce arthritic inflammation and angiogenesis in rats, and may attenuate and improve the pathological process of arthritis. In addition, the results revealed the involvement of LOX in diseases of the joint and angiogenesis in rats of the model group. This suggested that LOX inhibition may be a potential therapeutic target in such diseases.

The MMP family is a group of zinc-dependent endopeptidases that are present in the normal human body, degrade almost all extracellular matrixes, and are major regulators of tissue remodeling and extracellular matrix degradation $(25,26)$. High expression levels of MMP-2 (also known as gelatinase A) and MMP-9 (also known as gelatinase B) promote the occurrence and development of RA $(27,28)$. The expression levels of MMP-2 and MMP-9 are increased in the synovial membrane of patients with RA, which is a major causative factor of the degradation of extracellular matrix components, including collagen and proteoglycan, in the bones and cartilage of diseased joints (29-31). The results of the present study also demonstrated that the expression levels of MMP-2 and MMP-9 in the serum, synovial fluid and synovial membrane of rats in the model group were significantly higher compared with those in the control group, indicating that MMP-2 and MMP-9 are involved in the development of arthritis in rats of the model group. However, following the inhibition of LOX activity by BAPN, the expression levels of MMP-2 and MMP-9 in the serum, synovial fluid and synovial tissues in rats of the intervention group decreased to levels lower than those in the model and control groups. Pearson's correlation analysis revealed that LOX expression level in synovial tissues was positively correlated with MMP-2 and MMP-9 expression levels, suggesting that the inhibition of LOX may downregulate the expression of MMP-2 and MMP-9. The functionality of LOX in the attenuation or improvement of diseases in rats of the intervention group may be mediate by this mechanism. However, the specific pathway of MMP-2 and MMP-9 downregulation by LOX remains unclear. The detailed mechanism underlying the action of LOX in CIA rats requires further study.

Currently, there is no cure for RA. Hyperplasia of the synovial membrane and angiogenesis serve important roles in the occurrence and delay of RA; therefore, targeting this pathological basis in further investigation of potential therapeutic targets is expected to provide novel approaches for RA treatment (32). The results of the present study suggested that the inhibition of LOX may attenuate synovial hyperplasia and angiogenesis in type II collagen-induced CIA model rats and ameliorate the damage induced by MMP-2 and MMP-9.

In summary, the present study demonstrated high expressions levels of LOX in the synovial membranes, synovial fluid and serum of CIA rats. Inhibition of LOX attenuated inflammation, synovial hyperplasia, angiogenesis and expression of MMP-2 and MMP-9, indicating that LOX promotes synovial hyperplasia and angiogenesis in CIA rats. Therefore, LOX may be a potential target for the treatment of RA.

\section{Acknowledgements}

The present study was supported by the National Natural Science Foundation of China (grant. no. 81260459) and the Science and Technology Research Project of the Department of Education in Ningxia (grant. no. NGY2012050).

\section{References}

1. Mclnnes IB and Schett G: The pathogenesis of rheumatoid arthritis. N Engl J Med 365: 2205-2219, 2011.

2. Bansback NJ, Regier DA, Ara R, Brennan A, Shojania K, Esdaile JM, Anis AH and Marra CA: An overview of economic evaluations for drugs used in rheumatoid arthritis: Focus on tumour necrosis factor-alpha antagonists. Drugs 65: 473-496, 2005.

3. Gibofsky A: Overview of epidemiology, pathophysiology, and diagnosis of rheumatoid arthritis. Am J Manag Care 18 (13 Suppl): S295-S302, 2012.

4. Scherer HU and Burmester GR: A clinical perspective of rheumatoid arthritis. Eur J Immunol 39: 2044-2048, 2009.

5. Kagan HM and Li W: Lysyl oxidase: Properties, specificity, and biological roles inside and outside of the cell. J Cell Biochem 88: 660-672, 2003

6. Khakoo A, Thomas R, Trompeter R, Duffy P, Price R and Pope FM: Congenital cutis laxa and lysyl oxidase deficiency. Clin Genet 51: 109-114, 1997.

7. Li W, Zhou J, Chen L, Luo Z and Zhao Y: Lysyl oxidase, a critical intra- and extra-cellular target in the lung for cigarette smoke pathogenesis. Int J Environ Res Public Health 8: 161-184, 2011.

8. Rimar D, Rosner I, Nov Y, Slobodin G, Rozenbaum M, Halasz K, Haj T, Jiries N, Kaly L, Boulman N, et al: Brief report: Lysyl oxidase is a potential biomarker of fibrosis in systemic sclerosis. Arthritis Rheumatol 66: 726-730, 2014.

9. Mesarwi OA, Shin MK, Drager LF, Bevans-Fonti S, Jun JC, Putcha N, Torbenson MS, Pedrosa RP, Lorenzi-Filho G, Steele KE, et al: Lysyl oxidase as a serum biomarker of liver fibrosis in patients with severe obesity and obstructive sleep apnea. Sleep 38: 1583-1591, 2015.

10. Baiocchini A, Montaldo C, Conigiaro A, Grimaldi A, Correani V, Mura F, Ciccosanti F, Rotiroti N, Brenna A, Montalbano M, et al: Extracellular matrix molecular remodeling in human liver fibrosis evolution. PLoS One 11: e0151736, 2016.

11. Rankin EB and Giaccia AJ: Hypoxic control of metastasis. Science 352: 175-180, 2016.

12. Perryman L and Erler JT: Lysyl oxidase in cancer research. Future Oncol 10: 1709-1717, 2014.

13. Liu R, Sun B, Lin J, Song T, Li H, Wen P and Han M: Comparison of lysyI oxidase expression between active rheumatoid arthritis and active osteoarthritis. Chin J Rheumatol 17: 95-97, 2013 (In Chinese).

14. Rosloniec EF, Cremer M, Kang AH, Myers LK and Brand DD: Collagen-induced arthritis. In: Current Protocols in Immunology. Coico R and Shevach E (eds) John Wiley \& Sons, New York, 15.5.1-15.5.25, 2010 .

15. Elhai M,Chiocchia G, MarchiolC,Lager F, Renault G, Colonna M, Bernhardt G, Allanore Y and Avouac J: Targeting CD226/DNAX accessory molecule-1 (DNAM-1) in collagen-induced arthritis mouse models. J Inflamm 12: 9, 2015. 
16. Miana M, Galán M, Martínez-Martínez E, Varona S, JuradoLópez R, Bausa-Miranda B, Antequera A, Luaces M, MartínezGonzález J, Rodríguez C and Cachofeiro V: The lysyl oxidase inhibitor $\beta$-aminopropionitrile reduces body weight gain and improves the metabolic profile in diet-induced obesity in rats. Dis Models Mech 8: 543-551, 2015.

17. Gao HY, Luo J, Li XF, Lv Q, Wen HY, Song QZ, Zhao WP, Zhao XC, Zhang TT, Zhang SY and Zhi JM: Changes in focal adhesion kinase expression in rats with collagen-induced arthritis and efficacy of intervention with disease modifying anti-rheumatic drugs alone or in combination. Int J Clin Exp Pathol 8: 15573-15581, 2015.

18. Amdekar S and Singh V: Lactobacillus acidophilus maintained oxidative stress from reproductive organs in collagen-induced arthritic rats. J Hum Reprod Sci 9: 41-46, 2016.

19. Aciksari K, Yanar HT, Hepqul G, Ozucelik DN, Yanar F, Agcaoglu O, Eser M, Tanriverdi G, Topacoglu H, Ayvaci BM, et al: The effect of Beta-aminopropionitrile and prednisolone on the prevention of fibrosis in alkali esophageal burns: An experimental study. Gastroenterology Res Pract 2013: 574260, 2013.

20. Jutley G, Raza K and Buckley CD: New pathogenic insights into rheumatoid arthritis. Curr Opin Rheumatol 27: 249-255, 2015.

21. Haag S, Tuncel J, Thordardottir S, Mason DE, Yau AC, Dobritzsch D, Bäcklund J, Peters EC and Holmdahl R: Positional identification of RT1-B (HLA-DQ) as susceptibility locus for autoimmune arthritis. J Immunol 194: 2539-2550, 2015.

22. Klimiuk PA, Domysławska I, Sierakowski S and Chwiećko J: Regulation of serum matrix metalloproteinases and tissue inhibitor of metalloproteinases-1 following rituximab therapy in patients with rheumatoid arthritis refractory to anti-tumor necrosis factor blockers. Rheumatol Int 35: 749-755, 2015.

23. Mc Ardle A, Flatley B, Pennington SR and FitzGerald O: Early biomarkers of joint damage in rheumatoid and psoriatic arthritis. Arthritis Res Ther 17: 141, 2015.
24. Yue B: Biology of the extracellular matrix: An overview. J Glaucoma 23 (8 Suppl 1): S20-S23, 2014.

25. Visse R and Nagase H: Matrix metalloproteinases and tissue inhibitors of metalloproteinases: Structure, function, and biochemistry. Circ Res 92: 827-839, 2003.

26. Giannandrea M and Parks WC: Diverse functions of matrix metalloproteinases during fibrosis. Dis Model Mech 7: 193-203, 2014.

27. Pap T, Shigeyama Y, Kuchen S, Fernihough JK, Simmen B, Gay RE, Billingham M and Gay S: Differential expression pattern of membrane-type matrix metalloproteinases in rheumatoid arthritis. Arthritis Rheum 43: 1226-1232, 2000.

28. Elshabrawy HA, Chen Z, Volin MV, Ravella S, Virupannavar S and Shahrara S: The pathogenic role of angiogenesis in rheumatoid arthritis. Angiogenesis 18: 433-448, 2015.

29. Pu J, Fang FF, Li XQ, Shu ZH, Jiang YP, Han T, Peng W and Zheng CJ: Matrine exerts a strong anti-arthritic effect on type II collagen-induced arthritis in rats by inhibiting inflammatory responses. Int J Mol Sci 17: pii: E1410, 2016.

30. Zhou M, Qin S, Chu Y, Wang F, Chen L and Lu Y: Immunolocalization of MMP-2 and MMP-9 in human rheumatoid synovium. Int J Clin Exp Pathol 7: 3048-3056, 2014.

31. Xue M, McKelvey K, Shen K, Minhas N, March L, Park SY and Jackson CJ: Endogenous MMP-9 and not MMP-2 promotes rheumatoid synovial fibroblast survival, inflammation and cartilage degradation. Rheumatology (Oxford) 53: 2270-2079, 2014.

32. Itoh Y: Metalloproteinases: Potential therapeutic targets for rheumatoid arthritis. Endocr Metab Immune Disord Drug Targets 15: 216-222, 2015. 